\title{
Design of Intelligent Perception Module Based on Wireless Sensor Network and Basketball Sports Attitude
}

\begin{abstract}
Wei Jin
School of Physical Education, Taiyuan University of Technology, Taiyuan, Shanxi, China

Correspondence should be addressed to Wei Jin; jinwei@tyut.edu.cn

Received 9 January 2022; Revised 8 February 2022; Accepted 9 February 2022; Published 24 February 2022

Academic Editor: Xin Ning

Copyright (C) 2022 Wei Jin. This is an open access article distributed under the Creative Commons Attribution License, which permits unrestricted use, distribution, and reproduction in any medium, provided the original work is properly cited.

In recent years, along with microelectromechanical (MEMS) technique and wireless body area network (WBAN), wearable health monitoring systems have emerged. With sustainability posture detection technique, people pay more and more attention to mankind posture detection. Human posture detection technique has been widely used in medical, film and television, industry, sports, and other fields. Motion capture is a technique for measuring the motion of moving objects in three-dimensional space with great accuracy. It is the most efficient method for producing computer 3D animation and collecting human motion data. The use of motion capture systems in animation is becoming increasingly common. The problem of a single sensor having a large error in monitoring human motion and attitude is addressed. The use of multisensor data fusion technology is used to propose a human motion pattern recognition method based on data fusion of accelerometer and gyroscope. The system must effectively integrate the information of various sensors in order to achieve the goal of accuracy, timeliness, and reliability processing, and multisensor information fusion systems for various complex application objects are constantly emerging.
\end{abstract}

\section{Introduction}

Few smart products can provide basketball enthusiasts with visual data to help them find fun in daily training, so that daily basketball training is no longer boring, and can record, compare, and analyze some statistics on basketball [1]. Human movement gesture recognition has been widely used in sports competition, rehabilitation medicine, health monitoring, somatotransducery games, and other fields in recent years, thanks to the rapid development of key technologies such as man-machine interactive and wireless body area networks. In the study of movement detection techniques, acceleration transducers are commonly used. The measurement information is very accurate in the state of uniform speed change due to the working characteristics of the acceleration transducer. People's daily activities, on the other hand, are frequently variable acceleration movements, and the combination of gravitational and variable acceleration leads to measurement. The end result is completely incorrect. Dimension measurement, object positioning in the physical world, orientation determination, and computer and data processing are all part of the motion capture tech- nique [2]. By installing a specific tracker in the key parts of the moving object, the movement capture system captures its position and attitude, and after computer processing, it is saved as data that can be applied in animation production.

In the unconscious state of mankind body, the longterm, continuous, and dynamic information of various physiological information (such as ECG signal, blood oxygen saturation, respiratory rate, blood pressure, and body temperature) and motion information (including the speed and trajectory of mankind movement) of the mankind body can display, track, and warn the physiological health status of mankind body in real time, so as to achieve the purpose of daily monitoring and prevention. In order to solve the problem of a single movement signal, many researchers have proposed algorithms such as multitransducer fusion based on Kalman filtering, which fuses the attitude data of acceleration transducers, gyroscopes, and magnetoresistive transducers to obtain high-precision mankind attitude angles [3]. The use of information fusion techniques has gotten a lot of attention. The US has developed dozens of information fusion systems over the years, including some of the most well-known target detection and 
battlefield management systems. The fusion system's difficulty and complexity also demonstrate the feasibility of information fusion. Data fusion collects raw data or signals and estimates and predicts the presence and detectability of objects based on data associations and characteristics at the signal or pixel level [4]. It is a generalization and application of classical signal detection theory. The data and information that a fusion system receives can be at a single level of abstraction or multiple levels of abstraction. Multisource information fusion has become an important key technical means for a large number of military and civilian high-tech systems and is a hot topic in the current research field, thanks to the emergence of a large number of new transducers and estimates that are more complete and accurate. Multiple transducers are frequently used to measure the same target parameters at different locations because multitransducer data fusion makes use of the complementarity of multiple information to improve the quality of information. Overall, the use of a single technique for situation estimation is decreasing, and various movements and technologies can be layered on top of each other and used in tandem to improve estimation results [5]. The use of multiple movements in combination has become a research hotspot. Although a multitransducer system has many benefits, it is more expensive than a single transducer, and physical characteristics such as device size, weight, and power consumption all increase. To make a trade-off in practical applications, both benefits and drawbacks should be considered [6].

This paper uses the multitransducer data fusion means to discuss. Information fusion is a new information processing means for the specific problem of a system composed of multiple transducers of the same or multiple types [7]. It is also called multisource correlation, multisource synthesis, transducer mixing, or multitransducer. Fusion has a broader term of multitransducer information fusion or information fusion. Multitransducer information fusion refers to the multilevel, multifaceted, and multilevel processing of data from multiple transducers to generate new meaningful information, which cannot be obtained by any single transducer. A single transducer can no longer meet the needs of modern combat systems and must use multiple transducers to locate and detect targets.

\section{Related Work}

According to Reference [8], a machine learning algorithm can accurately identify four types of user actions (running, walking, cycling, and sitting) using an iPhone and Nike+iPod Sport Kit (naive Bayesian network). This system, however, requires users to insert the Nike+iPod Sport Kit chip into the sole, which is more limited in practice. Reference [9] proposes using only the data from the three-axis acceleration transducer to recognize five behaviors (walking, limping, jogging, going upstairs, and going downstairs) on Android mobile phones. The use of multiorder Markov chains to predict where users will arrive next was proposed in Reference [10], and it was discovered that second-order Markov chains had the best recognition effect. Reference [11] proposes a method for identifying activities in the home environment, and experiments show that a large number of small transducers with only simple state transition functions can identify these activities. A model for activity recognition using cheap, reliable, and low-power movement transducers is proposed in Reference [12]. Wearable transducers were proposed as a detection method for activity recognition in Reference [13]. These movements, on the other hand, are all centralized solutions that cannot solve potential detection errors caused by network delays nor meet real-time requirements. As a result, researchers began to investigate distributed movements. A distributed probabilistic model for identifying daily activities was proposed in Reference [14], but the definition of activities in this way is limited to the triggering sequence of transducers and requires a large amount of labeled data. Reference [15] proposed the concept of activity-based layering and proposed a distributed model to identify daily activities. However, when defining the activity model, only the sequence of activities was considered, and the duration was not considered, so a certain accuracy was lost. Reference [16] proposed an association graphbased mechanism to identify abnormal activities in home environments, but since this mechanism ignores activity duration information, it only works well in specific settings. Reference [17] proposed an abnormal activity detection means based on a simple transducer, but this means also did not consider the effect of duration on the model establishment, and the accuracy decreased to a certain extent. Reference [18] proposed a detailed classification scheme for the swimming propulsion mode of fish according to the different body parts used for swimming propulsion. Reference [19] proposed the "two-dimensional wave plate theory" through the subsurface flow theory and linearized boundary conditions, which treats fish as a thin plate and is used to analyze the hydrodynamic characteristics of bonito fish. Reference [20] proposed to use multiple transducers to build a mankind action recognition system, which can recognize postures such as sitting, lying, and standing and actions such as climbing stairs and running. Reference [21] proposed a general information fusion model and introduced the multilayer processing viewpoint of information fusion.

The argumentation means adopted in this paper is the data fusion means. Since a single data fusion algorithm has certain limitations, the advantage integration of two or more data fusion algorithms can effectively reduce the uncertainty of the system, environmental interference, and the impact of failure data on state estimation. Estimation is a high-level key information technique in an information fusion system. At the same time, multitransducer data fusion can also suppress the influence of transducer drift and noise to a certain extent.

\section{Gesture Recognition and Implementation}

3.1. General Overview of the System. Pose segmentation is important because it is the first step in the pose recognition process. This paper focuses on the recognition of threedimensional dynamic gestures using a movement transducer [22]. The goal is to create a gesture recognition algorithm based on the data collected by the movement transducer in 


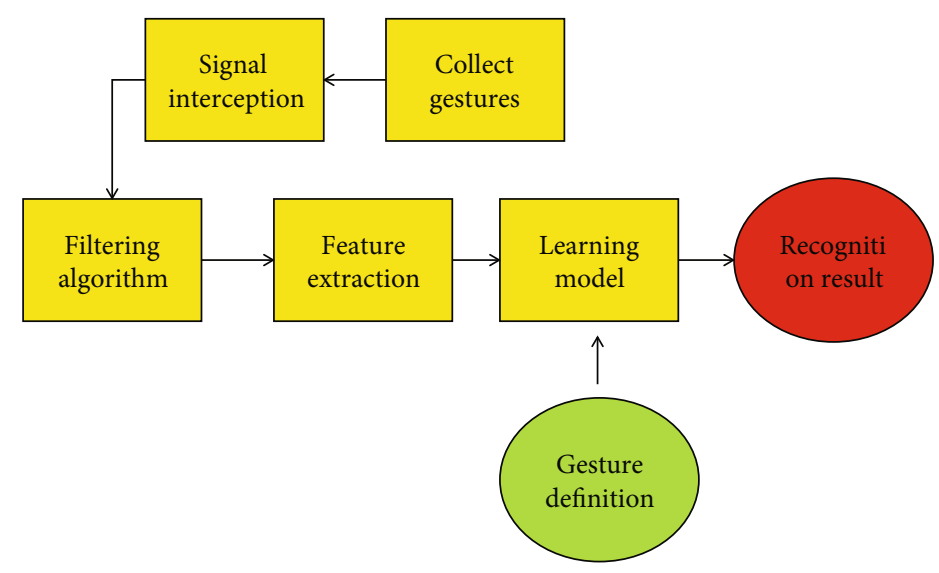

Figure 1: Flowchart of the gesture recognition system.

order to achieve quick and accurate gesture recognition. The definition of gesture varies due to the diversity and ambiguity of gesture, the passage of time and space, and the influence of various cultural backgrounds. Through literature research, it has been discovered that the use of inertial transducers to identify the basic movement of the mankind body has made significant progress; there are also many researches on dynamic posture based on inertial transducers when the mankind body is stationary; however, there is almost no research, the main reason being that the complexity of the basic movement of the mankind body causes significant interference to the dynamic posture signal, affecting the signal characteristics [23]. This topic is aimed at designing a set of three-dimensional dynamic gesture recognition systems from a macro perspective. The movement transducer collects information about the user's gesture movement at the input terminal. Following entry into the system, a series of calculations are carried out before the final gesture recognition result is displayed. Computer vision-based movements are used in the majority of current gesture recognition research, but they have an insurmountable problem [24]. Skin tones show clustering in the color space, allowing them to be distinguished from other colors, according to extensive experimental data. Skin color as a hand feature requires little calculation, has a quick processing speed, and is unaffected by factors like pose shape, size, and direction.

In modern society, with the advancement of science and technique and the improvement of people's material living standards, people hope that natural and intimate gesture interaction can be integrated into more technological products, bringing people a more comfortable operation and experience. Gesture recognition is to recognize the meaning expressed according to the user's gesture. It uses the mankind hand directly as the input device of the computer. The communication between the mankind and the computer will no longer require an intermediate medium. The user can simply define an appropriate gesture to the surrounding machines controlled. A complete pose needs to be divided into 3 stages, namely, preparation, action, and end, so it is necessary to identify the start and end points of the pose, which is also called pose cut in this paper. Existing research uses acceleration as an action feature for train- ing and recognition and has achieved a good recognition rate in the recognition of specific actions. However, there are shortcomings in the recognition of slow or rotationrelated movements. Skin-color-based pose segmentation also has some shortcomings; that is, it is difficult to adapt to complex backgrounds and changing lighting environments [25]. The effect of skin-color segmentation is poor, if not completely invalid, when skin-like areas appear in the background or the color of the light source is offset. The defined dynamic posture should be simple, practical, and obvious and should fit people's daily habits. Investigation of the gesture recognition system's most serious issues states that it is the core of the entire system to obtain accurate gesture data from the movement transducer module under the constraints of the environment, hardware configuration, capital, and other conditions. In general, interactive target recognition can be broken down into operations like switching and adjusting the size. On the one hand, realtime performance should be taken into account, and on the other hand, accuracy should be taken into account when judging the user's posture. To calculate the attitude angle of the transducer module, the data from the geomagnetic transducer and the acceleration transducer are combined in this paper. As a feature of the action [26], combine it with the acceleration data. It is necessary to create a library of prebuilt feature templates, which are then used to match each image position. There are many changes in nature, so we should try to extract the most important part, whether the posture is different or the same person. The data preprocessing, gesture modeling, and gesture recognition movements are all part of the gesture recognition system's system flow, as shown in Figure 1.

\subsection{Analysis of Multitransducer Data Fusion Problems}

3.2.1. Definition of Data Fusion. In this thesis, I use the multisensing movement. Let us talk about this method now. Along with computer and transducer techniques, multitransducer information fusion technique has gotten a lot of attention in recent years. The multitransducer information fusion technique is a hot topic in scientific research right now, and its goal is to figure out how to get more accurate 


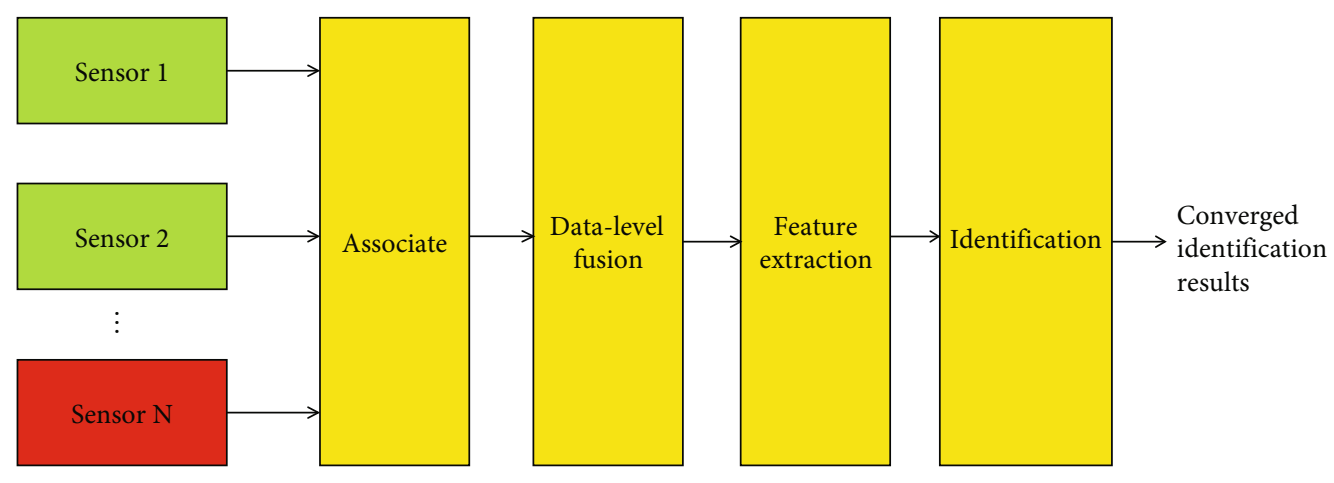

Figure 2: Data-level fusion.

estimation and reasoning by combining data from multiple sources rather than relying on a single one. The diversity and complexity of modern warfare are increasing as a result of the rapid development of the digital information society, particularly the development of computer, microelectronic, communication, and sensing techniques, and a single transducer can no longer guarantee that it can provide accurate and valid information [27]. Data fusion, also known as multisource correlation, multisource synthesis, transducer mixing, and other terms, is a new method of information processing for the problem of systems with multiple or multiple types of transducers. Data/information fusion with multiple transducers is referred to as data/information fusion. Data-level fusion is shown in Figure 2.

Multitransducer data fusion utilizes the complementarity of multiple information to improve the quality of information, so multiple transducers are often used to measure the same target parameters at different locations. Situation estimation is a high-level key technique in the information fusion system, and it is self-evident that the ability of realtime situation estimation to assist decision-making is of great significance to adapt to modern warfare. The complexity and diversification of information fusion and its applications make the content of data fusion to be studied very rich, and the basic theories covered are also very extensive. Situation estimation is the second-level fusion in the information fusion system, and it is one of the more active research technologies in this field. The emergence of multitransducer information fusion technique stems from the need for automatic fusion processing of multisource information in modern warfare. A human is one of the most complex and highly adaptive data fusion systems. Human eyes, ears, nose, tongue, and limbs are the transducers of sight, hearing, smell, taste, and touch. Information fusion processing models with four and five levels of processing can dynamically monitor the fusion processing process, optimize resource and transducer management, and feedback fusion result information in real time, ensuring that the fusion processing process is adaptive and achieves the best fusion effect. The diversity of information representation, the enormous amount of data, the complexity of information relationships, and the real-time nature of information processing in a multitransducer system far outnumber the human brain's comprehensive information processing capability. Because it extracts and processes the obtained information at a high level of abstraction; involves more parameters, factors, combat styles, and viewpoints; and is closer to the mankind thinking process, the situation estimation process is more complicated than the first-level fusion process. The brain will comprehensively process the information and make judgments about the surrounding environment when people obtain information about the surrounding environment through these transducers with various functions.

In recent years, the overall application of a single technique for situation estimation has gradually decreased, and various movements and technologies can be overlapped and used complementary to each other to obtain better estimation results. Information fusion was proposed in the early 1970s, and military applications were the origin of the technique. At present, there is no information fusion algorithm that can calculate all transducer fusion information [28]. In general, which algorithm to choose should be decided according to the specific application. Due to the wide range of application fields of data fusion theory, there are many kinds of models. The Data Fusion Group of the Joint Steering Committee of the US Department of Defense Laboratory gives a general model for the application of data fusion in the military field. The model started with three levels and later developed into four levels. Situation estimation technique is one of the most active research fields in information fusion. Since the mid-to-late 1970s to the present, many countries have carried out research on situation estimation in terms of theoretical systems and system implementation movements. Great progress has been made. Situation estimation is an important part of the command automation auxiliary decision-making system. It is based on military knowledge and military experience. It organically links the observed distribution of combat forces with activities and the surrounding environment of the battlefield, enemy combat intentions, and enemy mobility, determines the cause of the incident, and obtains an estimate of the enemy's force structure and use characteristics. Finally, a comprehensive situation map of the battlefield is formed. In fact, the cognitive process of mankind beings and other animals in nature to objective things is the fusion process of multisource information. In military systems, data fusion is mainly used in early warning systems, command and control of weapon systems, intelligence support systems, military force assessment and command systems, and space-ground integrated information fusion systems. 
3.2.2. Methods of Data Fusion. Since situation estimation is a high-level relationship extraction and processing of the obtained data flow, it is closer to the mankind thinking process and involves many factors and parameters, so it is more complicated than first-level fusion processing. The object of situation estimation in the real environment is the combat entity that is constantly moving and changing with the passage of time in the combat area. In fact, psychic estimation is enough to perceive and extract the psychic elements from such a moving object, in the process of knowing, understanding, and predicting. Although the existing methods provide a promising technical way for the practical application of situation estimation, the existing research results show that a single information fusion method is also difficult to work for the high-level fusion problem of situation estimation.

The following is a brief introduction to the commonly used fusion algorithms. The weighted fusion algorithm combines the redundant information from each transducer to the measurement data of the environmental characteristic parameters into an optimal estimate according to a certain weighting rule. In the situation estimation system, the detection of temporal relationship and spatial relationship is an important aspect of event detection. The information fusion system can be divided into three levels based on the data abstraction level: data-level fusion, feature-level fusion, and decision-level fusion. Various tracking algorithms are usually formed by combining data association algorithms with state estimation algorithms. Fuzzy logic is a mathematical tool for dealing with uncertainty in human cognition that can handle ambiguous semantic data. In a static environment, Bayesian inference is a rational mechanism for multitransducer information fusion. Continuous information fusion and relearning are carried out, a method for describing the information in neural networks as a probability distribution. When there is certain modeling and application of multitransducer information fusion, the means uses the system structure and uncertainty inference mechanism as a Bayesian estimation for each transducer of the neural network in the transducer, which provides good preevidence. When Bayesian estimation is used in an indirect way for fusion, different reasoning movements can be adapted for different situational frameworks depending on the knowledge representation and the representation of time, space, and causal constraints in subtasks (ideological potential). Traditional cluster analysis is a fixed classification, but because the boundaries between the states of each event object are not always clear, it is more appropriate to conduct the target cluster analysis using fuzzy mathematics.

\section{Intelligent Transducer Module Based on Basketball Posture}

\subsection{Data Characteristic Analysis Means}

4.1.1. Gyroscope Data Analysis. The gyroscope is one of the core measurement components of the inertial navigation system. Considering the engineering application, it is required to shorten the process of reaching the thermal equilibrium state after the gyroscope is started as much as possi- ble, so that the system can quickly enter the predetermined working state [29]. As an important inertial transducer, a gyroscope is the basic core device of the inertial navigation system (INS). From the liquid floating gyroscope in the 1950s to the dynamic tuning gyroscope and electrostatic gyroscope in the 1970s to the fiber optic gyroscope and ring laser gyroscope developed in the 1980s, the gyroscope has gone through roughly the following processes, to a micromachined gyroscope made using MEMS technology. The MEMS gyroscope, which first appeared in the second half of the 1980s, is characterized by its low cost, small size, long working life, wide dynamic range, ease of digitization, high intelligence, and strong antioverload capability. It is ideal for situations where there is a lot of movement and a lot of g. Microelectromechanical integration is simple to integrate and install into a variety of complex control systems. A constant zero offset and a noise signal make up the gyroscope static signal. The probability density distribution diagram shows that the noise is normally distributed, which is consistent with white noise signal characteristics. The static data diagram of the gyroscope is shown in Figure 3, and the probability density distribution diagram of the gyroscope is shown in Figure 4.

The gyroscope test and modeling are both done on a single gyroscope and are done at room temperature. The $N$ -point position means using a turntable are used to mark some gyroscope parameters. A satellite attitude measurement system consists of gyroscopes and optical transducers (such as infrared horizons, solar transducers, and star transducers) that can obtain continuous high-precision attitude and angular velocity information from satellites. This is a very important and broad application. Traditional gyroscopes used in space and military applications have rotating moving parts that are large, heavy, and consume a lot of power. The wear of high-speed rotating parts reduces their service life significantly, and it takes time to warm up the speed to the working speed. Traditional macroscopic gyroscopes are limited in their development due to stability and other issues. The standard deviation of the error tends to be stable as the value of $a$ decreases, and its value gradually increases. When the amplitude is small, there is a minimum value of $a=0.7$, which then increases, and the mean value of the error when the amplitude is large decreases with the decrease of slang, as shown in Figure 5.

The theory that studies the movement characteristics of gyroscopes is a branch of the dynamics of rigid bodies moving around a fixed point. In-depth study of the characteristics of the gyroscope should take its inertia as the basis. The gyroscope obtains the declination information through the angular velocity of the sensitive carrier relative to the inertial frame. With the bearing on the gyroscope frame shaft and the rotor of the gyroscope motor, the earliest application of the bearing is the rolling bearing. The static signal of the gyroscope obtained by the analysis of the original signal diagram contains constant components and noise components. From the statistical results, the statistical mean of the signal after Kalman filtering does not change much, and the mean square error is greatly reduced, but the effect is not as good as the first-order low-pass filtering of the 


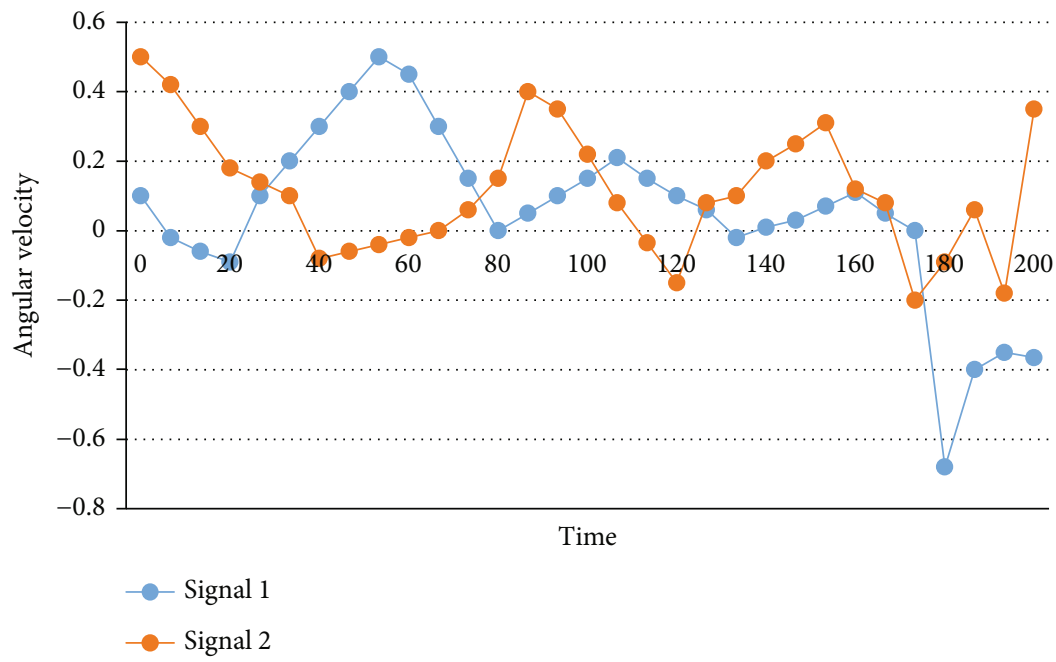

Figure 3: Gyrostatic data diagram.

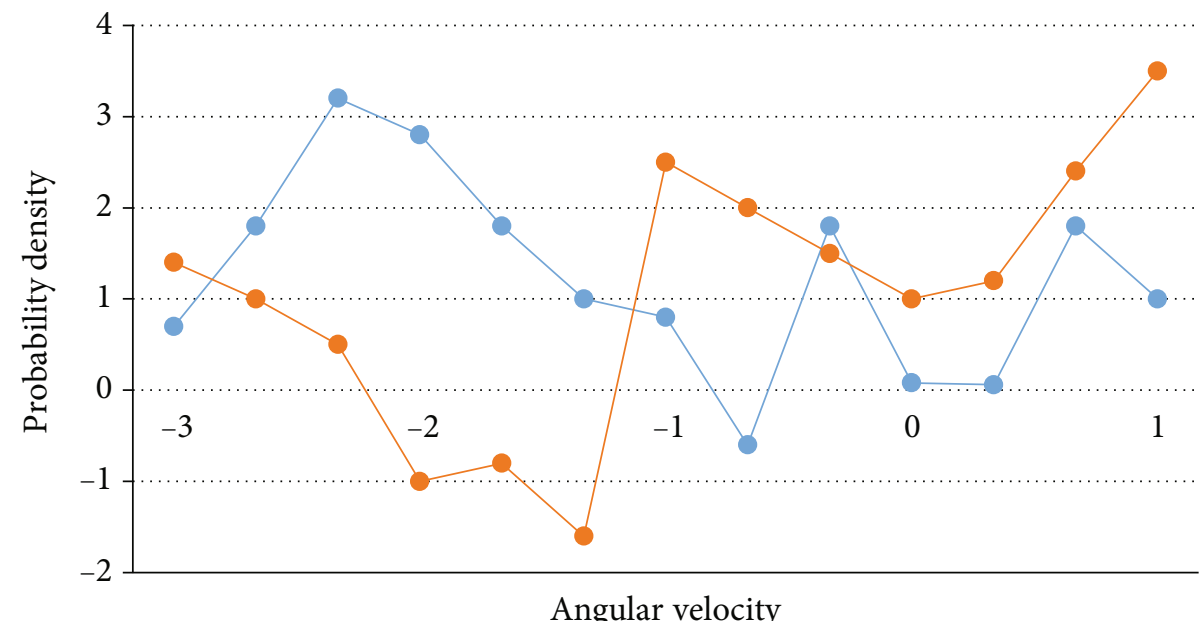

- Signal 1

$\longrightarrow$ Signal 2

Figure 4: Distribution of probability density of gyroscope.

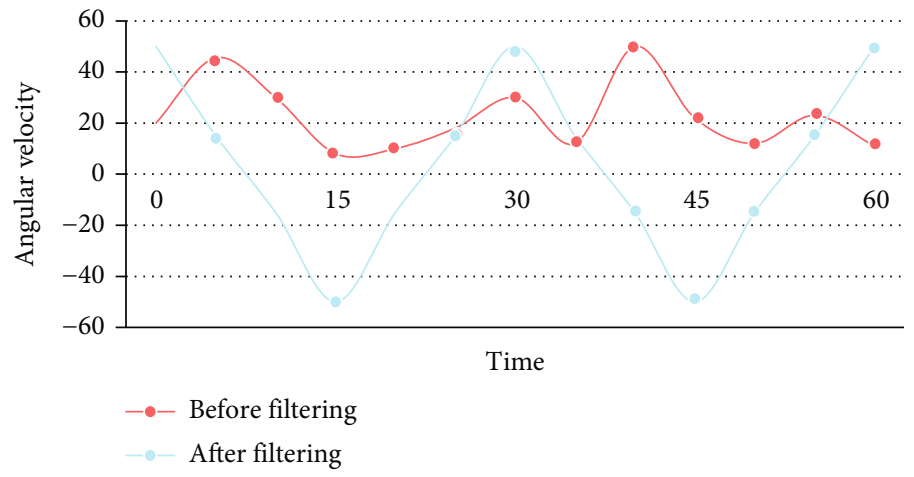

FIGURE 5: Swing test results (amplitude is $50^{\circ}$, sampling frequency is $100 \mathrm{~Hz}, a=0.7$ ). 


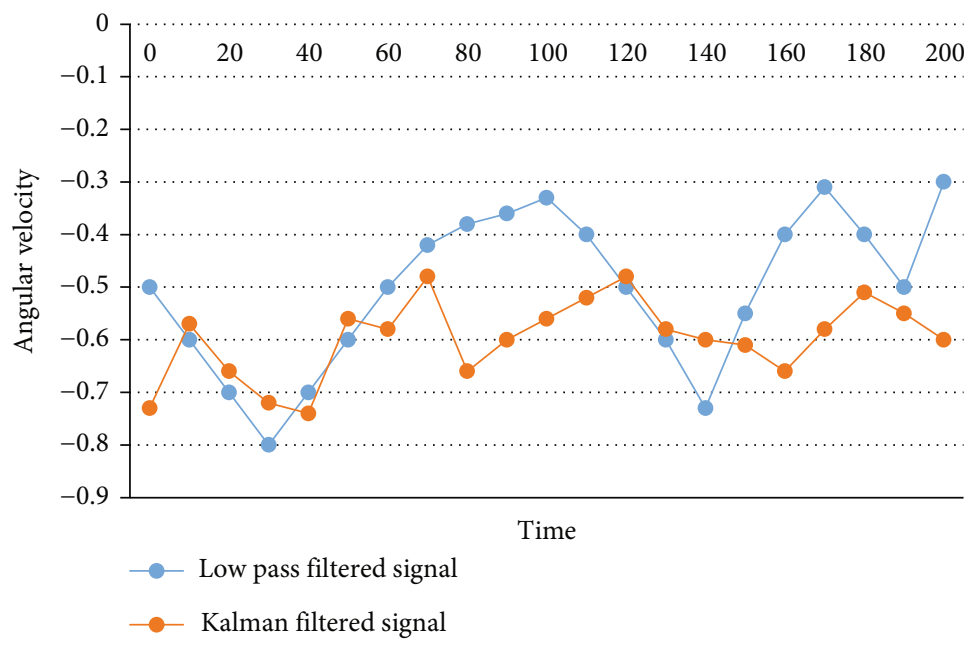

FIGURE 6: Kalman filtered data.

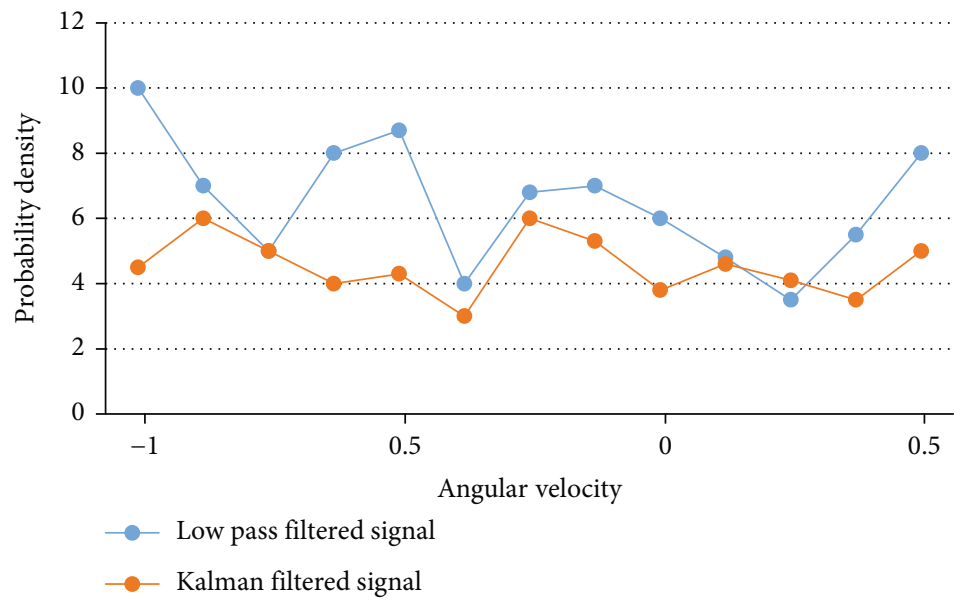

Figure 7: Kalman probability distribution.

optimal coefficient. The data after Kalman filtering is shown in Figure 6, and the Kalman probability distribution is shown in Figure 7.

Small and cheap requirements have promoted the development of microgyroscopes, which are widely used in tactical weapons, microaircraft, stable platforms, robots, military and civilian fields such as automobiles, surgical instruments, toys, computer mice, cameras, etc.

4.1.2. Acceleration Data Analysis. The movement speed is very important to the processing efficiency of the manufacturing system, and the change of the movement speed is due to the small acceleration of the system. The acceleration of the control system is not only related to the electromagnetic torque of the control system but also related to the rotational inertia and load torque of the controlled system. Silicon transducers, which combine the piezoresistive effect of silicon with integrated circuit technology, have high sensitivity, quick dynamic response, high precision, good stability, easy miniaturization, and mass production and are currently in widespread use. The three-dimensional acceleration design model is based on the string vibration accelerometer model from 1950. The mass is supported by three pairs of metal wires that are arranged vertically in space, and the acceleration is determined by the string's tensile stress or the modulation of the transverse resonance frequency. The electromechanical time constant of the system can be used to determine the speed change capability of the control system once the load has been determined. The smaller the constant, the higher the system's allowable acceleration. The piezoresistive effect of silicon was first studied in detail in the 1950 s by C.S. Smith, who determined that silicon has good elastic deformation properties and a significant piezoresistive effect. Since then, acceleration transducers have been used. The fiber optic acceleration transducer probe must have good directivity as a vector sensing probe. The current change speed of the servo device is represented by the electrical time constant of the control system, and the change of the current directly reflects the change of the electromagnetic torque, so the electrical time constant of the system affects the acceleration change speed.

The early accelerometers were semiconductor strain gauge pressure transducers and later developed into the 


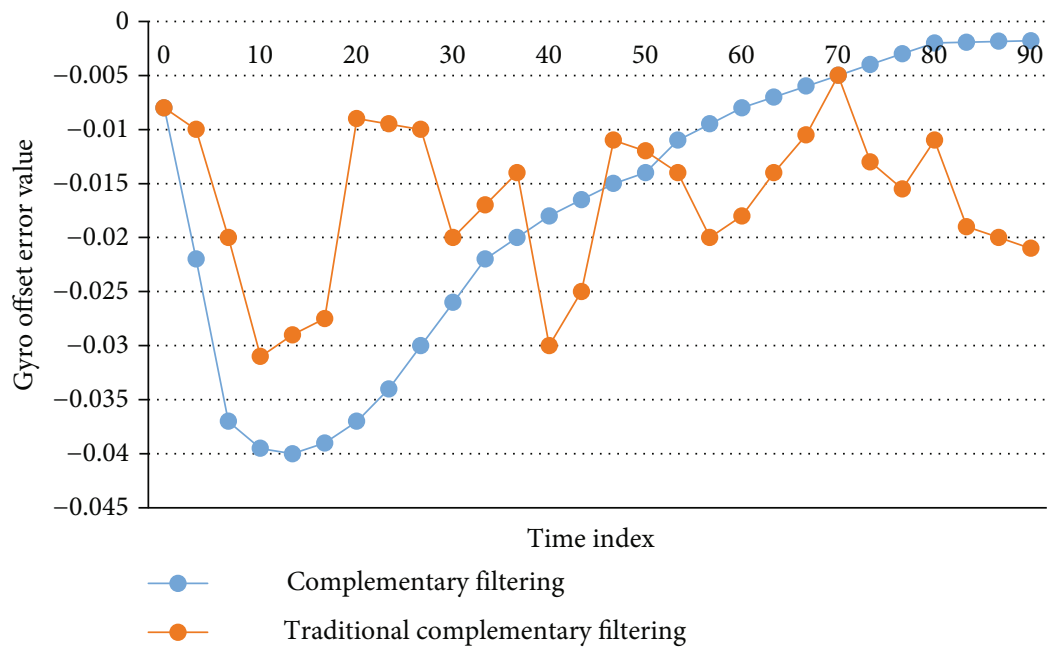

FIgURE 8: Gyro offset error.

use of diffused silicon movements to fabricate resistive strips on silicon wafers, called solid-state pressure transducers or diffused accelerometers. The transducer with a modular structure has good directivity and low crosstalk, which is the design means of most three-component acceleration transducers at present, but the structure design has a great influence on the final demodulation means and demodulation accuracy. In the design of the movement control system, a large acceleration margin can generally be guaranteed. If this point can be fully utilized, the average movement speed of the system can be significantly improved. Since the 1970s, the use of integrated circuit techniques to manufacture accelerometers has achieved rapid development, and a silicon cup-type diffusion pressure transducer with an integrated peripheral clamped resistance and a silicon diaphragm has been made. In order to ensure that the grating can accurately respond to the vibration of the mass block and facilitate the adjustment of the center wavelength of the grating, the transducer adopts bolt and beam structure adjustment.

\subsection{Several Common Attitude Calculation Movements}

\subsubsection{Attitude Calculation Based on Complementary} Filtering. Pose estimation has a wide range of applications such as aerial, underwater, robotics, navigation systems, games, industry, and augmented reality systems. Along with micromachine inertial technique, the use of micromachined gyroscopes, accelerometers, and magnetometers (MARGs) to construct miniature and low-cost heading and attitude systems has become one of the research hotspots in recent years. At this time, the most widely used attitude measurement technique is a strapdown inertial navigation systembased attitude calculation technique. We know that the gyroscope's dynamic response characteristics are better, but when the attitude is calculated, the cumulative error will increase as the integration time increases. When it comes to measuring attitude, the magnetometer and accelerometer have no cumulative error, but their dynamic response is much worse. Inertial measurement unit (IMU) devices, such as three-axis gyroscopes and three-axis accelerometers, are used to determine attitude. However, because of system noise and drift errors in the IMU transducer, which will accumulate over time, precise attitude control is difficult. Many well-established estimation movements have emerged from extensive research in the field of pose estimation, ranging from complex Kalman filters to simple complementary filters. The complementary filter algorithm can achieve a better estimation of the attitude, and the estimation accuracy is also better, as shown in the gyro offset error in Figure 8.

The attitude can be obtained by integrating the threeaxis gyroscope. The gyroscope has good dynamic response characteristics and high accuracy in the short term, but when it is used for attitude calculation, it will generate accumulated errors and eventually lead to attitude divergence. High-precision SINS systems are expensive and not suitable for many low-cost needs. We can use complementary filters to correct the data of the gyroscope, accelerometer, and magnetometer with each other, so that the measurement data is more accurate and the performance of the system is more stable. Adding PI-adjusted adaptive complementary filtering, the filtered signal is smoother, while the traditional complementary filtering does not filter high-frequency noise completely, as shown in Figure 9.

In general, the computational complexity of traditional pose or angle estimation filters is high. To this end, the research of satisfactory, accurate, and low computational complexity algorithms is the original intention of this paper. The accelerometer can be used for inclination measurement, but it is sensitive to gravity acceleration and movement acceleration at the same time, and the above attitude measurement system will produce a large measurement error when the carrier does a long-term maneuvering movement.

4.2.2. Extended Kalman Filter Attitude Solution Based on Gauss-Newton Iterative Method. The attitude calculation problem is to solve the direction cosine matrix of the current moment according to the measurement information at a certain moment. Due to the zero drift of the gyroscope, the gyroscope can be used to predict the state, and then, the 


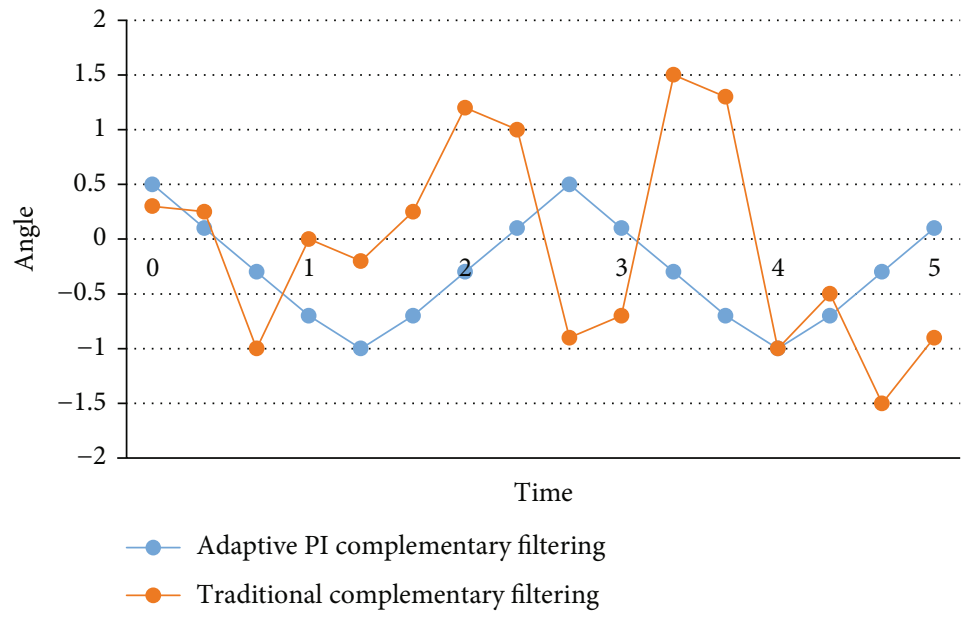

FIGURE 9: Comparison of traditional complementary filtering and adaptive PI complementary filtering.

accelerometer and the magnetometer can be used to update the predicted state to eliminate the accumulated error caused by the zero drift of the gyroscope. Kalman filtering is a filtering algorithm proposed by Kalman in 1960 to estimate the desired signal from the observations related to the extracted signal. The idea of the Kalman filter is to use statistical estimation to obtain the optimal estimation of the system state in the presence of random noise in the system under test and the measurement system. A set of different noise and drift-free attitude parameters can be obtained through the use of the accelerometer and magnetometer. The Kalman filter can properly balance the attitude parameters output by the gyro transducer with errors, by making full use of several different noise measurements and then estimating the attitude of the integrated navigation system to improve the accuracy of the navigation system, as shown in Figure 10.

Since the basic equation of Kalman filtering is a recursive form in the time domain, its calculation process is a continuous "prediction-correction" process that does not necessitate storing a large amount of data when solving and can be calculated whenever new data is observed. This filtering method is ideal for real-time processing and computer implementation. The Kalman filtering estimation of the state vector (unknown parameters) is unbiased and has a low variance. This method of estimation focuses on analyzing experimental data to estimate the system.

Let the state equation and observation equation of the estimated system be expressed by equations (1) and (2).

Equation of state:

$$
x_{k}=A x_{k-1}+B u_{k-1}+w_{k-1} \text {. }
$$

Observation equation:

$$
Z_{k}=H x_{k}+v_{k-1} \text {, }
$$

where $x$ is the state vector, $A$ is the state transition matrix, $B$ is the control matrix, $u$ is the system input vector, $Z$ is the measurement vector, and $H$ is the system observation matrix and is the process noise vector with zero mean and variance; the variance is zero; and the sum is uncorrelated.

The time update equation of the Kalman filter can be expressed as

$$
\begin{aligned}
& \widehat{x}_{\bar{k}}=A \widehat{x}_{k-1}+B u_{k-1}, \\
& \widehat{P} \bar{k}=A \widehat{p}_{k-1} A^{T}+Q .
\end{aligned}
$$

The state update equation of the Kalman filter can be expressed as

$$
\begin{aligned}
& K_{k}=P_{\bar{k}} H^{T}\left(H P_{\bar{k}} H^{T}+R\right)^{-1}, \\
& \widehat{x}_{k}=\widehat{x}_{\bar{k}}+K_{k}\left(z_{k}-H \widehat{x}_{\bar{k}}\right), \\
& P_{k}=\left(1-K_{k} H\right) P_{\bar{k}} .
\end{aligned}
$$

The current state variables are projected forward in time as a priori estimates to the measurement update equations, which correct the prior estimates to obtain a posteriori state estimates. When the time update equation and measurement update are finished, the measurement update equation calculates the Kalman filter gain, then the measurement output, and then, the state posterior estimation is generated according to equation (5); finally, the posterior covariance of the state is estimated according to equation (5). Following the equation, the entire process is repeated, with the posterior estimate from the previous calculation serving as the prior estimate for the subsequent calculation. One of the Kalman filter's most appealing features is its recursive inference.

The gyro transducer is a sensitive carrier angular movement element, because the gyro has errors in its manufacture; therefore, the expression of the actual output of the gyro is

$$
\omega_{b}^{\prime}=\omega_{b}+\varepsilon
$$

which is the ideal output of the gyro transducer and is the measurement error of the gyro element. The gyro 


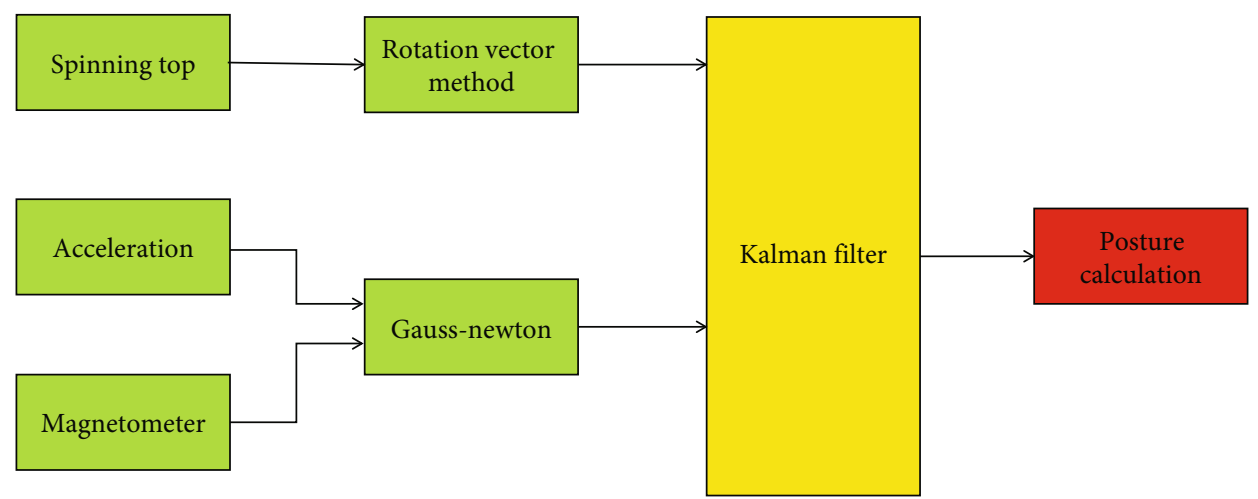

FIGURE 10: The working principle of the integrated navigation system.

measurement error generally includes installation error, scale factor error, and random error (i.e., gyro drift). Usually, the gyro drift model is taken as

$$
\varepsilon=\varepsilon_{b}+\varepsilon_{r}+w_{g}
$$

which is a random constant, is a first-order Markov process, is white noise, and is its variance. The mathematical model is

$$
\left\{\begin{array}{l}
\dot{\varepsilon}_{b}=0 \\
\dot{\varepsilon}_{r}=-\frac{1}{T_{r}} \varepsilon_{r}+w_{r}
\end{array}\right.
$$

which is the correlation time, is the driving white noise, and is its variance.

The traditional Kalman filter is ineffective for fractionalorder systems, and many projects include fractional-order cases. The fractional-order algorithm and Kalman filter are used to better apply the Kalman filter to the fractionalorder system. A new filtering algorithm, the fractional Kalman filtering algorithm, is created by combining algorithms. The influence of nongravity acceleration on attitude estimation is avoided by switching the extended Kalman attitude estimation algorithm to integrate the information of the micromachined gyroscope and the accelerometer, and a relatively accurate attitude angle and two-axis gyro bias are obtained.

\section{Conclusions}

Motion capture is a technique for precisely measuring the movement of moving objects in three-dimensional space, and it is the most efficient way to produce computer 3D animation and collect data on human movement. People have higher and higher expectations for the accuracy of mankind posture detection because it is widely used in many fields such as entertainment, medical treatment, and sports. In this paper, a mankind physiological parameter acquisition system based on the body transducer network is designed after extensive research and analysis of the characteristics of the body transducer network, with the goal of meeting some urgent needs of the elderly in physiological monitoring.
The micromechanical transducer includes a gyroscope, acceleration, and geomagnetic transducer, which compensates for gyroscope measurement errors and allows for more accurate capture of the human body's movement posture than a single angular velocity transducer. The mankind body posture detection system designed in this subject can detect mankind body posture after experiments and debugging. However, due to the broad range of knowledge and numerous technical difficulties involved in this subject, there are still many issues that need to be addressed, as well as some flaws. In future work, it must be improved and perfected.

\section{Data Availability}

The data used to support the findings of this study are included within the article.

\section{Conflicts of Interest}

The author does not have any possible conflicts of interest.

\section{References}

[1] E. Lopezosa-Reca, G. Gijon-Nogueron, I. Garcia-Paya, and A. B. Ortega-Avila, "Does the type of sport practised influence foot posture and knee angle? Differences between footballers and swimmers," Research in Sports Medicine, vol. 26, no. 3, pp. 345-353, 2018.

[2] Z. A. Abro, C. Hong, N. Chen, Y. Zhang, R. A. Lakho, and S. Yasin, "A fiber Bragg grating-based smart wearable belt for monitoring knee joint postures," Textile Research Journal, vol. 90, no. 3-4, pp. 386-394, 2020.

[3] Y. L. Kuo and S. F. Liu, "The foot posture index between elite athletic and sedentary college students," Kinesiology, vol. 49, no. 2, pp. 202-207, 2017.

[4] T. Wong and B. Paton, "Foot posture and its relationship with knee kinematics in barefoot running of the healthy adult," British Journal of Sports Medicine, vol. 51, no. 4, pp. 411.141411, 2017.

[5] R. S. Melo, A. R. Tavares-Netto, A. Delgado, C. C. Wiesiolek, K. M. Ferraz, and R. B. Belian, "Does the practice of sports or recreational activities improve the balance and gait of children and adolescents with sensorineural hearing loss? A systematic review," Gait \& Posture, vol. 77, pp. 144-155, 2020. 
[6] T. Wren, B. O’Callahan, M. J. Katzel et al., "Movement variability in pre-teen and teenage athletes performing sports related tasks," Gait \& Posture, vol. 80, pp. 228-233, 2020.

[7] Y. Jiang, T. Xia, L. Shen et al., "Facet-dependent $\mathrm{Cu}_{2} \mathrm{O}$ electrocatalysis for wearable enzyme-free smart sensing," ACS Catalysis, vol. 11, no. 5, pp. 2949-2955, 2021.

[8] H. Chen, H. Zhang, T. Xu, and J. Yu, "An overview of micronanoswarms for biomedical applications," ACS Nano, vol. 15, no. 10, pp. 15625-15644, 2021.

[9] A. Sadeqi, H. Rezaei Nejad, F. Alaimo, H. Yun, M. Punjiya, and S. R. Sonkusale, "Washable smart threads for strain sensing fabrics," IEEE Transducers Journal, vol. 18, no. 22, pp. 91379144, 2018.

[10] B. Żuk, M. Sutkowski, S. Paśko, and T. Grudniewski, "Posture correctness of young female soccer players," Scientific Reports, vol. 9, no. 1, article 11179, 2019.

[11] C. Ma, W. Li, R. Gravina, and G. Fortino, "Posture detection based on smart cushion for wheelchair users," Transducers, vol. 17, no. 4, p. 719, 2017.

[12] Z. Q. Yao, Y. Dai, Q. N. Li, D. Xie, and Z. H. Liu, “A novel posture positioning method for multi-joint manipulators," IEEE Transducers Journal, vol. 20, no. 23, pp. 14310-14316, 2020.

[13] R. M. Kwasnicki, G. W. V. Cross, L. Geoghegan et al., "A lightweight sensing platform for monitoring sleep quality and posture: a simulated validation study," European Journal of Medical Research, vol. 23, no. 1, p. 28, 2018.

[14] P. Li, N. Su, Z. Wang, and J. Qiu, " $\mathrm{A} \mathrm{Ti}_{3} \mathrm{C}_{2} \mathrm{~T}_{\mathrm{x}} \mathrm{MXene-based}$ energy-harvesting soft actuator with self-powered humidity sensing and real-time motion tracking capability," ACS Nano, vol. 15, no. 10, pp. 16811-16818, 2021.

[15] V. S. Dasari, B. Kantarci, M. Pouryazdan, L. Foschini, and M. Girolami, "Game theory in mobile crowdsensing: a comprehensive survey," Transducers, vol. 20, no. 7, p. 2055, 2020.

[16] O. Banos, H. Hermens, C. Nugent, and H. Pomares, "Smart sensing technologies for personalised e-coaching," Sensors, vol. 18 , no. 6 , p. $1751,2018$.

[17] I. A. Papistas and V. F. Pavlidis, "Contactless heterogeneous 3D ICs for smart sensing systems," Integration, vol. 62, pp. 329340, 2018.

[18] Y. T. Quek, W. L. Woo, and T. Logenthiran, "Smart sensing of loads in an extra low voltage DC pico-grid using machine learning techniques," IEEE Transducers Journal, vol. 17, no. 23, pp. 7775-7783, 2017.

[19] J. Han, W. Song, A. Gozho et al., "LoRa-based smart IoT application for smart city: an example of human posture detection," Wireless Communications and Mobile Computing, vol. 2020, Article ID 8822555, 15 pages, 2020.

[20] A. Jalal, I. Akhtar, and K. Kim, "Human posture estimation and sustainable events classification via pseudo-2D stick model and K-ary tree hashing," Sustainability, vol. 12, no. 23, p. $9814,2020$.

[21] D. B. Berry, B. Shahidi, and E. Ana, "Lumbar muscle structure predicts operational postures in active-duty marines," Journal of Orthopaedic and Sports Physical Therapy, vol. 48, no. 8, pp. 613-621, 2018.

[22] A. Jafarnezhadgero, S. Heshmatizadeh, S. Salahi-Movasagh, and F. Saki, "The timing pattern of selected muscles in male children with forward head posture compared to healthy control ones during running," Sports Biomechanics, vol. 5, no. 1, pp. 38-49, 2020.
[23] K. Hajduk and D. Schmidtbleicher, "Reliability of photographic postural assessment in male elite junior soccer players (under 16, 17 \& 19)," British Journal of Sports Medicine, vol. 51, no. 4, pp. 326.2-32326, 2017.

[24] S. Zhang, P. McCullagh, H. Zheng, and C. Nugent, "Situation awareness inferred from posture transition and location: derived from smartphone and smart home sensors," IEEE Transactions on Human-Machine Systems, vol. 47, no. 6, pp. 814-821, 2017.

[25] H. Ohi, H. Iijima, T. Aoyama, E. Kaneda, K. Ohi, and K. Abe, "Association of frontal plane knee alignment with foot posture in patients with medial knee osteoarthritis," BMC Musculoskeletal Disorders, vol. 18, no. 1, p. 246, 2017.

[26] C. D'Anna, M. Schmid, and S. Conforto, "Linking head and neck posture with muscular activity and perceived discomfort during prolonged smartphone texting," International Journal of Industrial Ergonomics, vol. 83, no. 7, p. 103134, 2021.

[27] Y. H. Chang, N. Sahoo, J. Y. Chen, S. Y. Chuang, and H. W. Lin, "ROS-based smart walker with fuzzy posture judgement and power assistance," Sensor, vol. 21, no. 7, p. 2371, 2021.

[28] R. Moreira, A. Teles, R. Fialho et al., "Can human posture and range of motion be measured automatically by smart mobile applications?," Medical Hypotheses, vol. 142, article 109741, 2020.

[29] R. Bootsman, P. Markopoulos, Q. Qi, Q. Wang, and A. A. A. Timmermans, "Wearable technology for posture monitoring at the workplace," International Journal of Human-Computer Studies, vol. 132, pp. 99-111, 2019. 